\title{
ANN model for prediction of powder packing
}

\author{
Mucahit Sutcu*, Sedat Akkurt ${ }^{1}$ \\ Mechanical Engineering Department, Izmir Institute of Technology, 35430 Izmir, Turkey
}

Available online 19 May 2006

\begin{abstract}
A multilayer feed forward backpropagation (MFFB) learning algorithm was used as an artificial neural network (ANN) tool to predict packing of fused alumina powder mixtures of three different sizes in green state. The data used in model construction were collected by mixing and pressing powders with average particle sizes of 350,30 and $3 \mu \mathrm{m}$ and with narrow particle size distributions. The data sets that were composed of green densities of cylindrical pellets were first randomly partitioned into two for training and testing of the ANN models. Based on the training data an ANN model of the packing efficiencies was created with low average error levels (3.36\%). Testing of the model was also performed with successfully good average error levels of $3.39 \%$.
\end{abstract}

(C) 2006 Elsevier Ltd. All rights reserved.

Keywords: Pressing; Porosity; $\mathrm{Al}_{2} \mathrm{O}_{3}$; Artificial neural network (ANN)

\section{Introduction}

The packing of particles has always been of interest to ceramists because a good packing particle compact will fire to higher densities. Such increases in densities result from reduction of diffusion distances and attainment of a larger number of particle-to-particle contacts. The resulting fraction of pores within the fired product is small. Therefore, it is important that ceramics are formed from well-packing mixtures of particles. The works on packing of particles may be divided into two categories: the discrete particle size distributions and the continuous particle size distributions.

Furnas's work deals with discrete particle size distributions for the packing of discrete particles sizes. ${ }^{1}$ He states that best packing occurs when finer particles exactly fill the space within the larger particles. When the size classes are three, the finest particles must fill the void space within the medium sized particles which themselves fill the spaces between the larger sized particles. The size ratio of coarse to fine particles ideally should be infinitely large to optimize packing. In real systems, however, ratios like 20:1 or 10:1 are common. The larger the ratio the closer it is to the theory that explains packing of discrete particles. ${ }^{1}$ It is, on the other hand, difficult to produce perfect

\footnotetext{
* Corresponding author. Tel.: +90 232750 7809; fax: +90 2327506505.

E-mail addresses: mucahitsutcu@iyte.edu.tr (M. Sutcu), sedatakkurt@iyte.edu.tr(S. Akkurt).

1 Tel.: +90 232750 6192; fax: +902327506505.
}

monodispersions to use in such systems. Since monodispersions are difficult to achieve, most of the studies deal with discrete size classes like one bounded by adjacent sieves. The fraction of complete particle size distributions that is bounded between two sieves is in fact a continuous distribution but it is nevertheless the closest to a monodispersion in process systems.

Another contribution to particle packing came from Westman and Hugill who developed an algorithm that used discrete theory of packing. ${ }^{2}$ They demonstrated a procedure for mixtures of two and three different sizes and gave an algorithm for higher number of size classes. McGeary proposed a discrete approach to packing of particles that were used as nuclear fuel pellets. $^{3}$

Andreassen's work, ${ }^{4}$ however, constitutes the basis for continuous particle size distributions where all particle sizes are present. He concluded that an appropriate packing theory should be one developed specifically for continuous distributions. Andreassen ${ }^{4}$ described the theory for the derivation of his equation from dimensional analysis and geometry. Granulation images of particles of two neighboring size classes were employed. Irrespective of the magnification, the same image was observed that led him to the particle size distribution equation for particle packing:

$$
\frac{\mathrm{CPFT}}{100 \%}=\left(\frac{D}{D_{\mathrm{L}}}\right)^{n}
$$

where CPFT is the cumulative percent finer than the size $(D)$, and $D_{\mathrm{L}}$ is the largest particle size. Andreassen also confirmed 
the validity of his equation via experimental tests and concluded that the distribution modulus, $n$ should be between 0.33 and 0.5 to obtain a dense packing.

Dinger and Funk later recognized that there should be a smallest particle size for packing equation of Andreassen. ${ }^{5}$ Therefore, they offered the following equation:

$\frac{\mathrm{CPFT}}{100 \%}=\frac{D^{n}-D_{\mathrm{S}}^{n}}{D_{\mathrm{L}}^{n}-D_{\mathrm{S}}^{n}}$

where $D_{\mathrm{S}}$ is the size of the smallest particle. The densest packing was calculated to occur when $n$ is equal to 0.37 . Real powders analyzed for distributions are well known to show bending of the CPFT versus log size curves. Hence, Dinger and Funk equation is closer to real distributions. In many discussions on packing of real particles, however, Andreassen's distribution provides a good fit. An example is the packing of refractory ceramics, which have a large size difference between neighboring size classes.

Zheng and Reed worked on the improved equations of the continuous particle size distribution for dense packing. ${ }^{6}$ These equations provide a method to study a continuous particle size distribution according to the principle of the Furnas model, which takes the volume fraction of fine and coarse particles as the function of their pore fractions for densest packing without considering the particle shape. Zou et al. has recently worked on the packing of mono-sized and multi-sized mixtures of wet coarse spheres. ${ }^{7,8}$ Their results indicated that porosity was strongly affected by particle size, their distribution and moisture content. Guerin et al. worked on the prediction of commercial alumina powder compact density using ANNs but the powders used in their study were not fused and hence not fully dense. ${ }^{9}$ Another study on packing prediction was done by Silva et al. who used statistical experimental design techniques to create a response surface of the particle compacts. ${ }^{10}$ So, to the knowledge of the authors, no study has yet been reported on prediction of powder compact densities using fully dense fused particles.

Neural network simulator algorithms for the prediction of percent porosities in three different sized fused alumina powder mixtures are developed in this study. ANNs are extensively used to model complex systems in a wide range of fields. ${ }^{11,12}$ The computational details about the ANN model construction are well documented in the literature. ${ }^{12}$ Therefore, such information will not be presented here.

\section{Data collection}

Abrasive grade fused alumina powders of three different sizes were obtained from Treibacher Co., Austria. ${ }^{13}$ The particle shapes and sizes are shown in Fig. 1. As can be seen in Fig. 1, the powders were all very dense and fused with little amount of pores. The size distributions of the particles were narrow because these powders were obtained from an abrasive manufacturer that has to meet strict limitations for sizes (FEPA). ${ }^{14}$ A number of blends ( $15 \mathrm{~g}$ each) were prepared from the above powders as follows (Fig. 2). Nine grams of distilled water was added to $15 \mathrm{~g}$ of powder mixture and the suspension was stirred

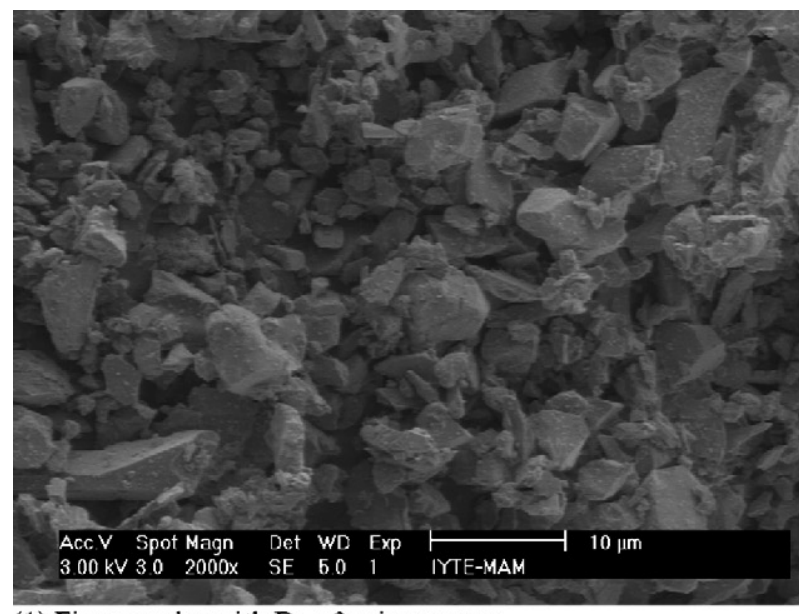

(1) Fine powder with $\mathrm{D}_{50}=3$ micrometers

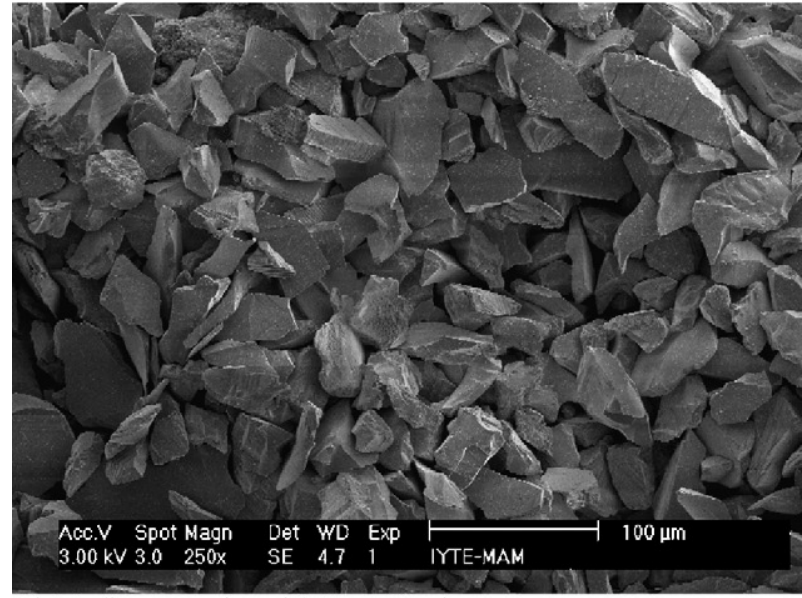

(2) Medium powder with $\mathrm{D}_{50}=30$ micrometers

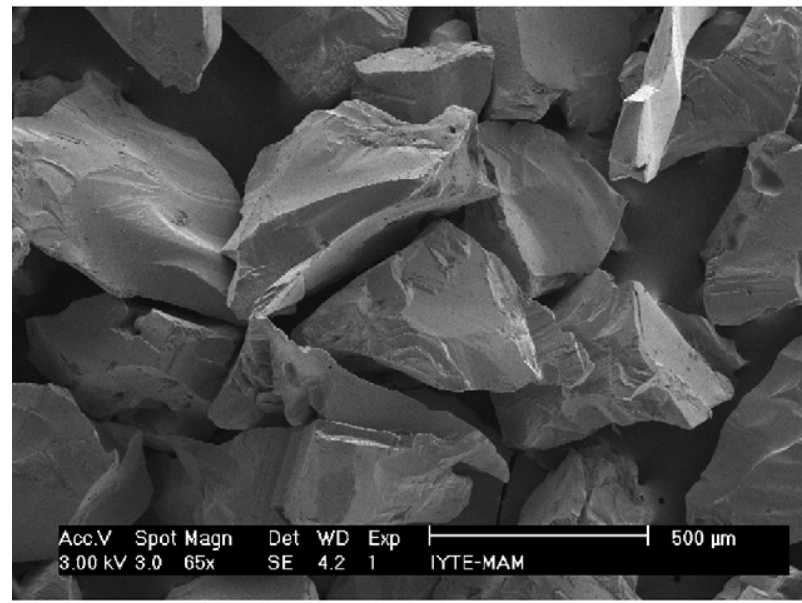

(3) Coarse powder with $D_{50}=350$ micrometers

Fig. 1. SEM image of the powders mixed in this study.

by a magnetic stirrer for $2 \mathrm{~h}$. After oven drying at $110^{\circ} \mathrm{C}$ for $2 \mathrm{~h}$ agglomerates were broken in mortar and pestle before addition of 20 drops of 5\% PVA solution and 3-5 ml of ethyl alcohol. The powder mixtures were then dried in oven, homogenized by using mortar and pestle, and pressed at $100 \mathrm{MPa}$ in the form of cylindrical pellets of $15 \mathrm{~mm}$ diameter and $6-10 \mathrm{~mm}$ height. Height and diameter of pellets were measured with a precision 


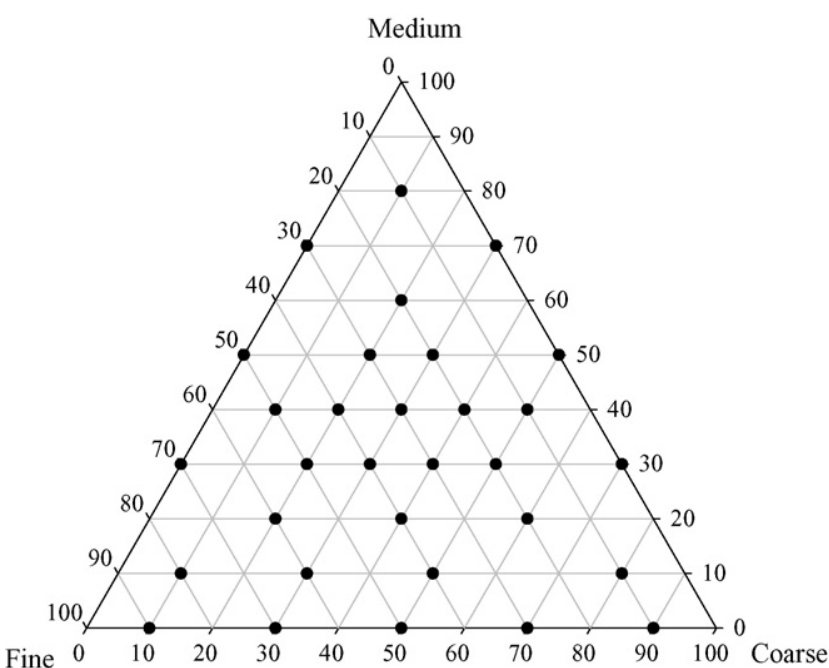

Fig. 2. Triangular plot of powder mixtures. calliper (Mitutoyo CD-15CP) and the porosities were calculated (Table 1).

\section{Model construction}

In this study, a three layer feed forward ANN architecture was constructed. In the input layer, there were three neurons for the three input variables. In the hidden layer, three neurons were chosen by trial and error. Finally, in the output layer, one neuron was used for the output variable of percent porosity. The input variables were as follows:

$x_{1}$ : percent coarse (powder code: F46); $x_{2}$ : percent medium (powder code: F320); $x_{3}$ : percent fine (powder code: F1200); $y$ : percent porosity.

Neurons in each layer were fully connected to every single neuron in the neighbouring layers. No bias term was utilized during modelling but a momentum term was used to help obtain

Table 1

The data that was used for the creation of the ANN model and the results of testing of the ANN model

\begin{tabular}{|c|c|c|c|c|c|c|c|}
\hline Blend code & $\begin{array}{l}\% \text { Coarse, F46 } \\
\text { D50 = 350 }\end{array}$ & $\begin{array}{l}\% \text { Medium, } \\
\text { F320 D50 = } 30\end{array}$ & $\begin{array}{l}\% \text { Fine, F1200 } \\
\text { D50 = } 3\end{array}$ & $\begin{array}{l}\text { Measured } \\
\text { porosity, } \%\end{array}$ & $\begin{array}{l}\text { ANN predicted } \\
\text { porosity, } \%\end{array}$ & $\begin{array}{l}\text { MIX } 10 \text { predicted } \\
\text { porosity, } \%\end{array}$ & $\begin{array}{l}\text { PAAE }^{\mathrm{a}} \text { for } \\
\text { ANN model }\end{array}$ \\
\hline \multicolumn{8}{|c|}{ PAAE for training $=3.36 \%$} \\
\hline 4 & 40 & 40 & 20 & 28.8 & 28.4 & 30.6 & 1.4 \\
\hline 44 & 70 & 30 & 0 & 31.9 & 31.2 & 34.6 & 2.1 \\
\hline 20 & 50 & 0 & 50 & 32.8 & 32.6 & 32.5 & 0.6 \\
\hline 42 & 30 & 70 & 0 & 37.4 & 37.3 & 27.4 & 0.2 \\
\hline 62 & 10 & 0 & 90 & 44.3 & 43.3 & 22.9 & 2.2 \\
\hline 25 & 70 & 0 & 30 & 28.9 & 29.5 & 34.6 & 2.0 \\
\hline 34 & 20 & 20 & 60 & 35.9 & 34.9 & 21.1 & 2.8 \\
\hline 55 & 10 & 10 & 80 & 40.9 & 40.2 & 21.8 & 1.8 \\
\hline 6 & 50 & 10 & 40 & 29.4 & 28.4 & 32.5 & 3.5 \\
\hline 12 & 50 & 40 & 10 & 27.2 & 28.1 & 32.5 & 3.3 \\
\hline 22 & 20 & 30 & 50 & 35.1 & 32.9 & 21.1 & 6.2 \\
\hline 47 & 0 & 30 & 70 & 31.6 & 34.7 & 20.4 & 9.9 \\
\hline 7 & 40 & 20 & 40 & 29.2 & 28.8 & 30.6 & 1.4 \\
\hline 48 & 30 & 0 & 70 & 38.6 & 40.7 & 27.4 & 5.5 \\
\hline 14 & 20 & 50 & 30 & 29.8 & 32.7 & 21.1 & 9.6 \\
\hline 15 & 30 & 50 & 20 & 31.9 & 31.3 & 27.4 & 1.9 \\
\hline 23 & 10 & 40 & 50 & 32.9 & 33.4 & 17.1 & 1.5 \\
\hline 39 & 0 & 70 & 30 & 34.9 & 34.4 & 25.0 & 1.5 \\
\hline 10 & 50 & 30 & 20 & 26.4 & 26.6 & 32.5 & 0.7 \\
\hline 17 & 60 & 20 & 20 & 24.7 & 26.2 & 33.7 & 6.2 \\
\hline 24 & 0 & 50 & 50 & 35.8 & 33.6 & 19.0 & 6.0 \\
\hline \multicolumn{8}{|c|}{ PAAE for testing $=3.39 \%$} \\
\hline 29 & 20 & 60 & 20 & 33.0 & 33.7 & 23.9 & 2.3 \\
\hline 3 & 30 & 40 & 30 & 30.3 & 30.4 & 27.4 & 0.4 \\
\hline 51 & 10 & 80 & 10 & 40.4 & 37.2 & 27.3 & 8.0 \\
\hline 8 & 30 & 30 & 40 & 30.5 & 30.6 & 27.4 & 0.3 \\
\hline 9 & 20 & 40 & 40 & 32.1 & 32.4 & 21.1 & 0.8 \\
\hline 1 & 40 & 30 & 30 & 27.8 & 27.9 & 30.6 & 0.4 \\
\hline 54 & 90 & 0 & 10 & 33.8 & 34.4 & 35.8 & 1.8 \\
\hline 18 & 50 & 50 & 0 & 32.9 & 31.8 & 32.5 & 3.3 \\
\hline 33 & 30 & 10 & 60 & 36.0 & 36.1 & 27.4 & 0.3 \\
\hline 49 & 80 & 10 & 10 & 25.8 & 30.0 & 35.3 & 16.3 \\
\hline
\end{tabular}

Porosities of pellets were obtained in green state. Porosities predicted by MIX10 software are also included.

a PAAE: percent average absolute error. 


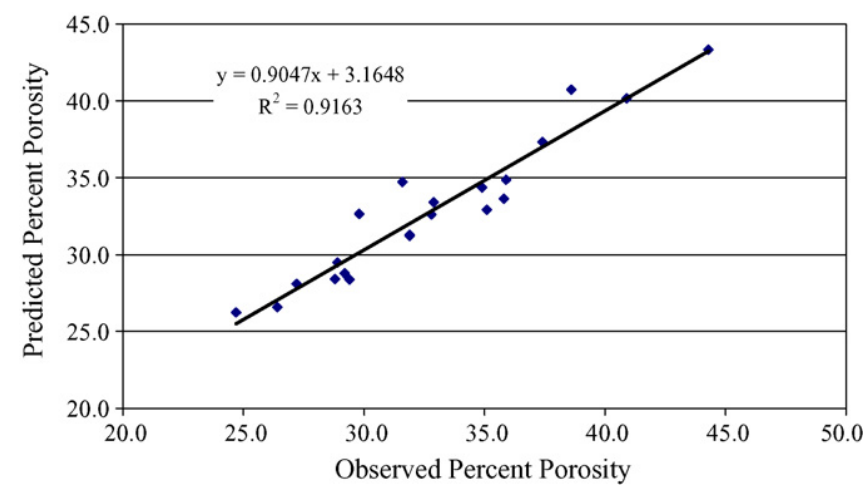

Fig. 3. Observed vs. predicted values of the percent porosity for training of the ANN model.

faster convergence during iterations. There were a total of 31 data sets 21 of which were used for the training of the ANN and the remaining 10 were for the testing of the model. Each data set had four components $\left(x_{1}, x_{2}, x_{3} ; y\right)$, three of which were the input variables while the fourth one was the output variable. The program operated for 20,000 iterations and the optimal weights were calculated with an percentage average absolute error (PAAE) for learning of $3.36 \%$ for percent porosity (Table 1; Fig. 3). The $r^{2}$ value for learning was 0.92 . The model was then tested with physically measured data using the remaining 10 data sets. The results of testing runs are also given in Table 1 and Fig. 4, where the low magnitude of percentage error of $3.39 \%$ (or $r^{2}=0.85$ ) indicated that the testing was successfully performed. Because of the limited number of experimental data only ten testing outputs were compared to real measured data. Large test data sets are always preferred when possible. As can be seen in Table 1 and Figs. 3 and 4, the performance of the model was quite satisfactory. More data is being collected and the work is in progress for three component powder mixing systems.

The individual powder size distributions employed in this study were not truly monodispersions but were narrow size distributions. Attempts were also made to predict the density of the three component mixtures using Dinger and Funk's MIX10 software. ${ }^{15}$ But the results obtained from the software did not provide good predictions with the measured data in this study (Table 1). Dinger suggested that these discrepancies resulted from the excessively large number of interparticle frictions caused by the fine sized particles in the compact. ${ }^{5}$ These frictions apparently interrupted the movements of the fine particles

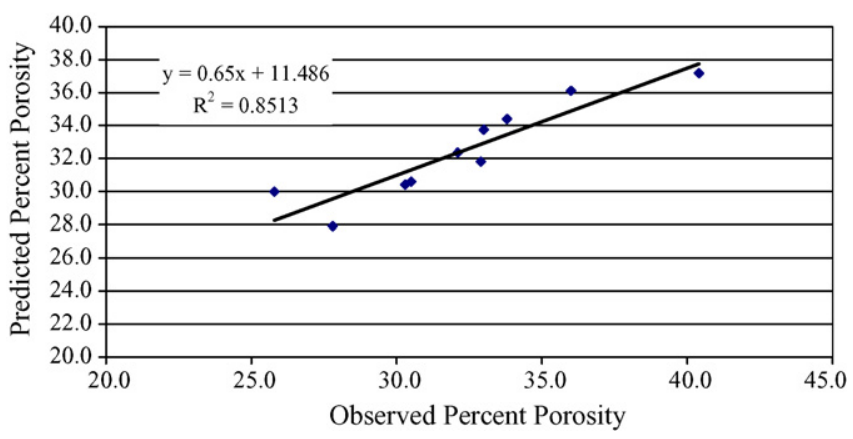

Fig. 4. Observed vs. predicted values of the percent porosity for testing of the ANN model. to fit in their expected void spaces between the larger particles as was the assumption in Westman and Hugill's algorithm. Dinger also proposed that MIX10 software was not developed to handle narrow size distributions like in this study.

\section{Conclusions}

In this study, an ANN model was developed for the prediction of percent porosity in three component powder mixtures. The satisfactory predictions of the observed percent porosities by the model indicated that ANN could be a useful tool for modelling such powder mixtures. A comparison of the ANN model was made to MIX10 predictions and it was found that MIX10 was poor in predicting porosities in mixtures of powder that are distributed in a narrow size range. Fused powders used in this study were manufactured primarily for use as a grinding material. Therefore, they had narrow size distributions due to specifications required by users. MIX10 was therefore not effective in packing prediction in this study. The ANN model could be utilized by engineers to locate the best packing mixture combination.

\section{References}

1. Furnas, C. C., Reletions Between Specific Volume, Voids and Size Composition in Systems of Broken Solids of Mixed Sizes, No. 2894, U.S. Bureau of Mines Report of Investigations, 1928.

2. Westman, A. E. R. and Hugill, H. R., The packing of particles. Journal of American Ceramic Society, 1930, 13(10), 767-779.

3. McGeary, R. K., Mechanical packing of spherical particles. Journal of American Ceramic Society, 1961, 44, 413-422.

4. Andreassen, A. H. M. and Andersen, J., Ueber die Beziehung zwischen Kornabstufung und Zwischenraum in Producten aus Losen Koernern (Mit Einigen Experimenten). Kolloid-Z, 1930, 50, 217-228.

5. Dinger, D. R. and Funk, J. E., Predictive Process Control of Crowded Suspensions. JEFCO Ceramic Consultants, Seneca, 1993.

6. Zheng, J., Johnson, P. F. and Reed, J. S., Improved equation of the continuous particle size distribution for dense packing. Journal of American Ceramic Society, 1990, 73(5), 1392-1398.

7. Zou, R. P., Xu, J. Q., Feng, C. L., Yu, A. B., Jonston, S. and Standish, N., Packing of multi-sized mixtures of wet coarse spheres. Powder Technology, 2003, 130, 77-83.

8. Zou, R. P., Feng, C. L. and Yu, A. B., Packing density of binary mixtures of wet spheres. Journal of American Ceramic Society, 2001, 184(3), 504-508.

9. Guerin, V., Prédiction et compréhension de la densification des poudres commerciales d'alumine et de fer grâce à une approche par réseau de neurones artificiels. Ph.D. Thesis. EPFL, Switzerland, 2004.

10. Silva, A. P., Segadaes, A. M. and Devezas, T. C., Optimization of the packing density of alumina powder distributions using statistical techniques. Ceramica, 2004, 50(October/December(316)) [Sao Paulo].

11. Akkurt, S., Ozdemir, S., Tayfur, G. and Akyol, B., The use of GA-ANNs in the modeling of compressive strength of cement mortar. Cement and Concrete Research, 2003, 33, 973-979.

12. Munakata, T., Fundamentals of the New Artificial Intelligence Beyond Traditional Paradigms. Springer-Verlag, New York, 1998.

13. Treibacher Co., Fused Alumina Products Catalogue, Austria, 2005, http://www.treibacher-schleifm.com/bonded.html.

14. Federation of European Producers of Abrasives, FEPA Powder Size Specifications, 2005, http://www.fepa-abrasives.org.

15. Funk, J. E. and Dinger, D. R., Predictive Process Control of Crowded Particulate Suspensions Applied to Ceramic Manufacturing. Kluwer Academic Publishers, Boston, MA, 1994. 